CERN-TH/2001-377

\title{
Considerations on Super Poincaré Algebras and their Extensions to Simple Superalgebras
}

\author{
S. Ferrara ${ }^{\star}$ and M. A. Lledó ${ }^{\text {. }}$ \\ * CERN, Theory Division, CH 1211 Geneva 23, Switzerland, \\ INFN, Laboratori Nazionali di Frascati, Italy and \\ Department of Physics and Astronomy, University of California, Los Angeles, U.S.A. \\ † INFN, Sezione di Torino, Italy and \\ Dipartimento di Fisica, Politecnico di Torino, \\ Corso Duca degli Abruzzi 24, I-10129 Torino, Italy
}

\begin{abstract}
We consider simple superalgebras which are a supersymmetric extension of the spin algebra in the cases where the number of odd generators does not exceed 64. All of them contain a super Poincaré algebra as a contraction and another as a subalgebra. Because of the contraction property, some of these algebras can be interpreted as de Sitter or anti de Sitter superalgebras. However, the number of odd generators present in the contraction is not always minimal due to the different splitting properties of the spinor representations under a subalgebra. We consider the general case, with arbitrary dimension and signature, and examine in detail particular examples with physical implications in dimensions $d=10$ and $d=4$.
\end{abstract}




\section{Introduction}

Super Poincaré algebras [1] are non semisimple superalgebras [2, [3, 团. Their even part is the Poincaré algebra (plus some extra generators that we will see below) and their odd part carries one or more (for $N$-extended supersymmetry) spinor representations of the underlying Lorentz algebra.

A spinor representation of the Lorentz algebra is an irreducible complex representation whose highest weights are the fundamental weights corresponding to the right extreme nodes in the Dynkin diagram. These are representations of the spin group that do not descend to representations of the orthogonal group.(For a review see Ref.[5, 间). We will call odd charges or spinor charges the generators of the odd part of the super Poincaré algebra. A reality condition must be imposed on the spinor charges to obtain a real Lie superalgebra.

Generically, we can write the anticommutator of two spinor charges as

$$
\left\{Q_{\alpha}^{I}, Q_{\beta}^{J}\right\}=\gamma_{\alpha \beta}^{\mu} p_{\mu} G^{I J}+\sum_{k} \gamma_{(\alpha \beta)}^{\left[\mu_{1} \cdots \mu_{k}\right]} Z_{\left[\mu_{1} \cdots \mu_{k}\right]}^{[I J]} .
$$

Here the indexes $\alpha$ run over the spinor representation, and $I, J=1, \ldots N$. $Z_{\left[\mu_{1} \cdots \mu_{k}\right]}^{[I J]}$ are even generators that are in a antisymmetric tensorial representation (a representation on the antisymmetric $n$th tensor product of the fundamental representation space) of the Lorentz group and commute with the translation generators.

In general there is a group $\mathrm{G}$ that acts on $Q^{I}$, which depends on the particular properties of the spinor representations in different signatures and dimensions. $G^{I J}$ is then an invariant tensor under this group and $Z^{I J}$ is in the two fold (symmetric or antisymmetric) representation of such group. It is called the automorphism group because its action leaves invariant the Lie superbrackets of the Poincaré superalgebra (it acts trivially on the other generators). The symmetry properties of $Z^{I J}$ are the same than the symmetry properties (in $\alpha, \beta)$ of $\gamma_{(\alpha \beta)}^{\left[\mu_{1} \cdots \mu_{k}\right]}$, which in turn depend only on the space-time dimension modulo 8 (and not on the signature).

From the bracket (1), we see that the even part of a super Poincare algebra has an abelian ideal that contains the spacetime translation generators and the $Z$ generators. Together with the odd charges they form a central extension of the supertranslation algebra. The physical interpretation of the $Z$ generators is related to p-brane charges [7, [8], that is, to certain configura- 
tions of supergravity theories in which the expectation value of the $Z$-charges is non zero.

Superconformal algebras are simple supersymmetric extensions of the conformal algebra. In Ref.9 these extensions for $N=1$ were studied for arbitrary dimension $(d=s+t)$ and signature $(\rho=s-t)$ and in Ref.[10] the case of extended supersymmetry was treated. There are in general two superconformal algebras, a maximal one which is always $\mathfrak{o s p}(1 \mid 2 N n)(2 n$ is the dimension of the spinor representation of the odd generators) and a minimal one, whose bosonic part is the Spin $(\mathrm{s}, \mathrm{t})$-algebra of the spinor representation. Only in dimensions $d=3,4,5,6$ is it possible to find a simple superalgebra with a bosonic part which factorizes as a direct sum of the orthogonal algebra $\mathfrak{s o}(s, t)$ plus a simple R-symmetry algebra [11], as required by the ColemanMandula theorem [12]. The odd part of the superalgebra is a direct sum spinor representations [13]. For higher dimensions, the bosonic part is also the direct sum of an R-symmetry subalgebra and a spacetime subalgebra, but the spacetime subalgebra is enlarged with extra generators.

In the web of connections between string theory and $\mathrm{M}$-theory [14, or possible generalizations as F-theory [15] or S-theory [16], it is natural to investigate the role played by the simple superalgebra, even in the cases $d>6$ [9, 10, 17, 18, 19, 20. As in the purely bosonic case, the super Poincaré algebra with $Z$-charges can be obtained from simple superalgebras in two different ways. One is by contraction [21], the other as sub-superalgebra [17, 18.

Let spacetime be a manifold of dimension $d$ with an (indefinite) metric of signature $(s, t)$. The Poincaré group acts on flat spacetime, $\mathbb{R}^{d} \simeq$ $\operatorname{ISO}(s, t) / \mathrm{SO}(s, t)$. Its Lie algebra, $\mathfrak{i s o}(s, t)$, is a subalgebra the conformal algebra of $\mathbb{R}^{d}$, the simple algebra $\mathfrak{s o}(s+1, t+1)$ [22]. Other possible backgrounds are the symmetric spaces $\mathrm{SO}(s+1, t) / \mathrm{SO}(s, t)$, with signature $(s, t)$. There is a contraction of the isometry algebra $\mathfrak{s o}(s+1, t)$ which gives the Poincaré algebra $\mathfrak{i s o}(s, t)$. Interchanging the roles of $s$ and $t$ we have a different contraction, from $\mathfrak{s o}(s, t+1)$. For physical signature, the two spaces are the de Sitter space $\mathrm{SO}(d, 1) / \mathrm{SO}(d-1,1)$ and the anti de Sitter one $\mathrm{SO}(d-1,2) / \mathrm{SO}(d-1,1)$. Unitary multiplets of the anti de Sitter superalgebra in dimension 11, osp $(1 \mid 32)$ where investigated in Ref.[23].

The super Poincaré algebra in dimension $d$ is a subalgebra of the superconformal algebra in the same dimension, appearing with different number extra generators in the abelian ideal, depending whether one looks at the minimal or the maximal superconformal algebra. Ref. 24] is an attempt to 
formulate M-theory as a spontaneously broken phase of its superconformal extension, where the symmetry under the superconformal algebra $\mathfrak{o s p}(1 \mid 64)$ is broken to the super Poincaré subalgebra with 2 and 5-brane charges.

Anti de Sitter and de Sitter superalgebras are supersymmetric extensions of $\mathfrak{s o}(d-1,2)$ and $\mathfrak{s o}(d, 1)$ respectively. They play an important role in the framework of the AdS and dS/CFT duality [25, 26, 27, 28].

Also, it is possible to obtain the super Poincaré algebra as a contraction of a simple superalgebra. We have the de Sitter and anti de Sitter superalgebras (simple extensions of the de Sitter and anti de Sitter algebras respectively), although not always the contraction of an $N=1$ super algebra gives an $N=1$ super Poincaré algebra. In Ref.19 the possibility of using some superalgebra gauge theory which gives M-theory as a particular low energy configuration (contraction) is explored.

Simple superalgebras embedding ordinary spacetime supersymmetry algebras are also relevant to explore how the theories depend upon the signature of space-time and provide a clue on the existence of supergravity theories with non lorentzian spacetime signature, as conjectured in Ref. [29] on the basis of time like $\mathrm{T}$ duality, with the $\mathrm{M}, \mathrm{M}^{\prime}$ and $\mathrm{M}^{*}$-theories in eleven dimensions.

The paper is organized as follows. In Section 2 we enumerate all the (minimal) superconformal algebras with 64,32, 16 and 8 spinor charges in dimensions $d=3, \ldots 11$ and arbitrary signature. We observe that the same superalgebra may be obtained from spacetimes with different signatures $\rho, \rho^{\prime}$ if they are congruent $\bmod 8, \rho= \pm \rho^{\prime}+8 n$, which may suggest a duality of the physical theories. In Section 3 the de Sitter and anti de Sitter superalgebras in dimensions $d=3, \ldots 12$ are considered and their contractions to super Poincaré algebras are studied. In Section 4 we consider physically interesting examples in $d=4$ and $d=10$. In the Appendix we give some basic definitions about Lie superalgebras.

\section{Super conformal algebras in diverse dimen- sions}

Superconformal algebras with up to 64 spinor real charges correspond to different real forms of complex superalgebras whose even part contains $\mathfrak{s o}(s+$ $1, t+1)$ and whose odd part is a direct sum of spinor representations of the same algebra. A spinor in dimension $d+2, d=s+t$, has complex dimension 
$2^{(d+1) / 2}$ for $d$ odd and $2^{d / 2}$ for $d$ even (chiral spinors). The dimension of the real representation depends on the reality condition (the same than the complex in the real case, twice the complex dimension in the quaternionic and complex case). In even dimension, when the superalgebra is of type $\mathfrak{s l}(m \mid n)(d+2=2,6)$, it contains left and right spinors (non chiral algebra) while if the algebra is of type $\mathfrak{o s p}(m \mid n)$ then it is chiral (in fact, the metric preserving condition halves the number of odd generators with respect to the linear superalgebra). For example, for $d=12$ the superconformal algebra is linear (or unitary) and for $N=1$ it has already 128 charges. So the maximal dimension that we can consider is $d=11$.

$d=11$. For $\rho=1,7 \bmod 8$ we have $\mathfrak{o s p}(1 \mid 64)$ with 64 odd charges. It corresponds to spacetimes of type $(10,1)$ (M-theory), $(9,2)\left(\mathrm{M}^{*}\right.$-theory) and $(6,5)$ (M'-theory) [29].

$d=10$. For $\rho=0 \bmod 8$, we have $\mathfrak{o s p}(2-q, q \mid 32),(q=0,1)$ with 64 charges and $\mathfrak{o s p}(1 \mid 32)$ with 32 charges. They correspond to spacetimes of type $(5,5)$ and $(9,1)$.

For $\rho=2,6 \bmod 8$ we have $\mathfrak{o s p}(1 \mid 32, \mathbb{C})$ with 64 odd charges and spacetimes of type $(6,4),(10,0)$ and $(8,2)$.

For $\rho=4$ we have $\mathfrak{o s p}\left(2^{*} \mid 16,16\right)$ with 64 charges and spacetime $(7,3)$.

These correspond to different forms of Type IIA, IIB and $(1,0)$ theories studied in Ref. 29.

$d=9$. For $\rho=1,7 \bmod 8$ we have $\mathfrak{o s p}(2-q, q \mid 32)(q=0,1)$ with 64 charges and $\mathfrak{o s p}(1 \mid 32)$ with 32 charges. They correspond to spacetimes of type $(9,0)$, $(5,4),(8,1)$.

For $\rho=3,5$ we have $\mathfrak{o s p}\left(2^{*} \mid 16,16\right)$ with 64 charges and spacetimes of type $(6,3)$ and $(7,2)$.

$d=8$. For $\rho=0 \bmod 8$ we have $\mathfrak{s l}(16 \mid 2)$ with 64 charges and $\mathfrak{s l}(16 \mid 1)$ with 32 charges. They correspond to spacetimes of types $(4,4)$ and $(8,0)$.

For $\rho=2,6$ we have $\mathfrak{s u}(8,8 \mid 2-q, q)(q=0,1)$ with 64 charges and $\mathfrak{s u}(8,8 \mid 1)$ with 32 charges. They correspond to spacetimes of type $(5,3)$ and $(7,1)$.

For $\rho=4 \bmod 8$ we have $\mathfrak{s u}^{*}(16 \mid 2)$ with 64 charges and spacetime of type $(6,2)$. 
$d=7$. For $\rho=1,7$ we have $\mathfrak{o s p}(8,8 \mid 4)$ with 64 charges and $\mathfrak{o s p}(8,8 \mid 2)$ with 32 charges. They correspond to spacetimes of type $(4,3)$ and $(7,0)$.

For $\rho=3,5$ we have $\mathfrak{o s p}\left(16^{*} \mid 4-2 q, 2 q\right)(q=0,1)$ with 64 charges and $\mathfrak{o s p}\left(16^{*} \mid 2\right)$ with 32 charges. They correspond to spacetimes of type $(5,2)$ and $(6,1)$.

$d=6$. For $\rho=0$ we have $\mathfrak{o} \mathfrak{s p}(4,4 \mid 8)$ with 64 charges, $\mathfrak{o} \mathfrak{s p}(4,4 \mid 4)$ with 32 charges and $\mathfrak{o s p}(4,4 \mid 2)$ with 16 charges. They correspond to spacetime of type $(3,3)$.

For $\rho=2,6$ we have $\mathfrak{o s p}(8 \mid 4, \mathbb{C})_{\mathbb{R}}$ with 64 charges and $\mathfrak{o s p}(8 \mid 2, \mathbb{C})$ with 32 charges. They correspond to spacetimes of type $(6,0),(4,2)$.

For $\rho=4$ we have $\mathfrak{o s p}\left(8^{*} \mid 8-2 q, 2 q\right)(q=0,1,2)$ with 64 charges, $\mathfrak{o s p}\left(8^{*} \mid 4-\right.$ $2 q, 2 q)(q=0,1,2)$ with 32 charges and $\mathfrak{o s p}\left(8^{*} \mid 2\right)$ with 16 charges. They correspond to spacetime of type $(5,1)$.

$d=5$. For $\rho=1$ we have $\mathfrak{o} \mathfrak{s p}(4,4 \mid 8)$ with 64 charges, $\mathfrak{o} \mathfrak{s p}(4,4 \mid 4)$ with 32 charges and $\mathfrak{o s p}(4,4 \mid 2)$ with 16 charges. They correspond to spacetime of type $(3,2)$.

For $\rho=3,5$ we have $\mathfrak{o s p}\left(8^{*} \mid 8-2 q, 2 q\right)(q=0,1,2)$ with 64 charges, $\mathfrak{o s p}\left(8^{*} \mid 4-\right.$ $2 q, 2 q)(q=0,1)$ with 32 charges and $\mathfrak{o s p}\left(8^{*} \mid 2\right)$ with 16 charges. They correspond to spacetimes of type $(5,0)$ and $(4,1)$.

For $d=5$ there exists a smaller superalgebra, the exceptional superalgebra $\mathfrak{f}_{4}^{p}$. The integer number $p$ denotes the real form of the complex superalgebra $\mathfrak{f}_{4}$, which depends on the signature of spacetime. For $\rho=3,5$ the even part of the superalgebra is $\mathfrak{s p i n}(7-p, p) \oplus \mathfrak{s u}(2)$ with $p=2,1$. In these signatures, the spinors are quaternionic (pseudoreal), so there exists a pseudoconjugation in the spinor space, which together with the pseudoconjugation in the fundamental of $\mathfrak{s l}(2, \mathbb{C})$ defining $\mathfrak{s u}(2)$ gives a conjugation defining the real form of the superalgebra. For signatures $\rho=1,7$ we have an even part $\mathfrak{s p i n}(7-p, p) \oplus \mathfrak{s l}(2, \mathbb{R})$, with $p=3,0$. In these cases the spinors are real, so the conjugation defining the real form of the superalgebra is formed with the conjugation in the spinor space and the conjugation in the fundamental of $\mathfrak{s l}(2, \mathbb{C})$ defining $\mathfrak{s l}(2, \mathbb{R})$. The superalgebra has 16 charges. (For more on conjugations, pseudoconjugations and real forms see Ref. [9]. See also Ref.[30] 
$d=4$. For $\rho=0$ we have $\mathfrak{s l}(4 \mid 8)$ with 64 charges, $\mathfrak{s l}(4 \mid 4)$ with 32 charges, $\mathfrak{s l}(4 \mid 2)$ with 16 charges and $\mathfrak{s l}(4 \mid 1)$ with 8 charges. They correspond to spacetime of type $(2,2)$.

For $\rho=2$ we have $\mathfrak{s u}(2,2 \mid 8-q, q)(q=0, \ldots 4)$ with 64 charges, $\mathfrak{s u}(2,2 \mid 4-q, q)$ $(q=0,1,2)$ with 32 charges, $\mathfrak{s u}(2,2 \mid 2-q, q)(q=0,1)$ with 16 charges and $\mathfrak{s u}(2,2 \mid 1)$ with 8 charges. They correspond to spacetime of type $(3,1)$.

For $\rho=4$ mod 8 we have $\mathfrak{s u}^{*}(4 \mid 8)$ with 64 charges, $\mathfrak{s u}^{*}(4 \mid 4)$ with 32 charges, $\mathfrak{s u}^{*}(4 \mid 2)$ with 16 charges and spacetime of type $(4,0)$.

$d=3$. For $\rho=1$ we have $\mathfrak{o s p}(16-q, q \mid 4)(q=0, \ldots 8)$ with 64 charges, $\mathfrak{o s p}(8-q, q \mid 4)(q=0, \ldots 4)$ with 32 charges, $\mathfrak{o s p}(4-q, q \mid 4)(q=0,1,2)$ with 16 charges, $\mathfrak{o s p}(2-q, q \mid 4)(q=0,1)$ with 8 charges and $\mathfrak{o} \mathfrak{s p}(1 \mid 4)$ with 4 charges. They correspond to spacetime of type $(2,1)$.

For $\rho=3$ we have $\mathfrak{o s p}\left(16^{*} \mid 2,2\right)$ with 64 charges, $\mathfrak{o s p}\left(8^{*} \mid 2,2\right)$ with 32 charges, $\mathfrak{o s p}\left(4^{*} \mid 2,2\right)$ with 16 charges and $\mathfrak{o s p}\left(2^{*} \mid 2,2\right)$ with 8 charges. They correspond to spacetime of type $(3,0)$.

\section{De Sitter and anti de Sitter superalgebras and their contractions}

We write the simple superalgebras that are extensions of de Sitter $(\mathfrak{s o}(d, 1))$ and anti de Sitter $(\mathfrak{s o}(d-1,2))$ algebras in physical signature $(d-1,1)$.

For $d=4,5,12$, the de Sitter superalgebra exists only with $N$ even.

In $d=6$ and $d=10$ the de Sitter and anti de Sitter superalgebras coincide. This is because the signature $\pm \rho$ of the the $(d+1)$-dimensional spaces (where the de Sitter or anti de Sitter algebras are linearly realized) are congruent modulo 8 ( 3 and 5 for $d=6,1$ and 7 for $d=10$ ). For anti de Sitter we have that the superalgebras of $d=6,7$ and $d=10,11$ coincide. For $d=6$ there exists a smaller subalgebra, the exceptional superalgebra $\mathfrak{f}_{4}$ with two real forms, $\mathfrak{f}_{4}^{1}$ and $\mathfrak{f}_{4}^{2}$ whose bosonic parts are $\mathfrak{s p i n}(6,1) \oplus \mathfrak{s u}(2)$ and $\mathfrak{s p i n}(5,2) \oplus \mathfrak{s u}(2)$ respectively. $\mathfrak{f}_{4}$ has a non chiral odd part of type $(1,1)$. They are the proper de Sitter and anti de Sitter superalgebras. For $d \leq 7$ one can find a simple superalgebra whose bosonic part has the de Sitter or anti de Sitter algebra as a factor. For higher dimensions this is not true, as one can check directly from Table 1 . 


\begin{tabular}{|c|l|c||l|c||c|}
\hline$d$ & de Sitter & odd & anti de Sitter & odd & odd SP \\
\hline 3 & $\mathfrak{o s p}(N \mid 2, \mathbb{C})_{\mathbb{R}}$ & $4 N$ & $\mathfrak{o} \mathfrak{s p}(N-q, q \mid 2)$ & $2 N$ & $2 N$ \\
4 & $\mathfrak{o} \mathfrak{s p}\left(N^{*} \mid 2,2\right)$ & $4 N$ & $\mathfrak{o} \mathfrak{s p}(N-q, q \mid 4)$ & $4 N$ & $4 N$ \\
5 & $\mathfrak{s u} \mathfrak{u}^{*}(4 \mid N)$ & $8 N$ & $\mathfrak{s u}(2,2 \mid N-q, q)$ & $8 N$ & $8 N$ \\
6 & $\mathfrak{o} \mathfrak{s p}\left(8^{*} \mid 2 N-2 q, 2 q\right)$ & $16 N$ & $\mathfrak{o} \mathfrak{s p}\left(8^{*} \mid 2 N-2 q, 2 q\right)$ & $16 N$ & $8 N$ \\
7 & $\mathfrak{o} \mathfrak{s p}(8 \mid 2 N, \mathbb{C})$ & $32 N$ & $\mathfrak{o} \mathfrak{s p}\left(8^{*} \mid 2 N-2 q, 2 q\right)$ & $16 N$ & $16 N$ \\
8 & $\mathfrak{o} \mathfrak{s p}(8,8 \mid 2 N)$ & $32 N$ & $\mathfrak{o} \mathfrak{s p}\left(16^{*} \mid 2 N-2 q, 2 q\right)$ & $32 N$ & $16 N$ \\
9 & $\mathfrak{s l}(16 \mid N)$ & $32 N$ & $\mathfrak{s u}(8,8 \mid N-q, q)$ & $32 N$ & $16 N$ \\
10 & $\mathfrak{o} \mathfrak{s p}(N-q, q \mid 32)$ & $32 N$ & $\mathfrak{o} \mathfrak{s p}(N-q, q \mid 32)$ & $32 N$ & $16 N$ \\
11 & $\mathfrak{o} \mathfrak{s p}(N \mid 32, \mathbb{C})_{\mathbb{R}}$ & $64 N$ & $\mathfrak{o} \mathfrak{s p}(N-q, q \mid 32)$ & $32 N$ & $32 N$ \\
12 & $\mathfrak{o} \mathfrak{s p}\left(N^{*} \mid 32,32\right)$ & $64 N$ & $\mathfrak{o} \mathfrak{s p}(N-q, q \mid 64)$ & $64 N$ & $64 N$ \\
\hline
\end{tabular}

Table 1: De Sitter and anti de Sitter superalgebras.

The contractions of these algebras to Poincaré superalgebras where studied in detail in Ref. 99 for $N=1$. It is of interest to note that the contractions give super Poincaré algebras with a number of odd generators which is not, in general, the minimal one in super Poincaré. In Table 1 we have added the dimension of the odd part of the superalgebra ("odd") together with the dimension of the odd part of the $N$-extended super Poincaré algebra for a spacetime of type $(d-1,1)$ ("odd SP"). The de Sitter superalgebra gives the correct contraction for $d=4,5,12$. The anti de Sitter superalgebra gives the correct contraction for $d=4,5,7,11,12$. The remaining case give twice the number of odd generators. For $d=6,10$ one obtains by contraction a non chiral algebra (In both, de Sitter and anti de Sitter), and the same happens if one makes the contraction of $\mathfrak{f}_{4}$. Physically, in dimension $d \leq 7$ supergravity theories exist with both, Minkowski and anti de Sitter supersymmetric solutions.

\section{Examples}

We consider some examples in $d=10$ and $d=4$.

Spacetime of type $(\mathbf{9 , 1})$. We have $d=2 \bmod 8$ and $\rho=0 \bmod 8$. The chiral spinor modules $S^{ \pm}$are real of dimension $2 n=16$. There is a chiral Poincaré superalgebra. The $N=2$ chiral super Poincaré algebra is the IIB 
algebra. One can also construct a non chiral one with the odd generators in the direct sum $S^{+} \oplus S^{-}$and it is the IIA algebra. Both have 32 odd spinor charges.

The conformal algebra is $\mathfrak{s o}(10,2)$. Poincaré superalgebras are subalgebras of the conformal superalgebras. The chiral algebra of type $(1,0)$ is a subalgebra of $\mathfrak{o s p}(1 \mid 32)$, the embedding given by

$$
\begin{array}{cc}
\mathfrak{s p}(32) & \stackrel{\supset}{ } \mathfrak{s o}(10,2) \\
32 & \longrightarrow S^{+}=32
\end{array}
$$

Type IIB algebra (type $(2,0))$ is a subalgebra of $\mathfrak{o s p}(2-q, q \mid 32)$, with $q=0,1$ and $q=0$ for compact R-symmetry. It has 64 spinor charges. Finally, this superalgebra is embedded (not as a subalgebra) into another superalgebra with 64 spinor charges, osp $(1 \mid 64)$, which has the interpretation of the superconformal algebra of a spacetime of type $(10,1)$ (that is, one dimension more).

The embedding of the even parts and decompositions of the representations which are the odd part are as follows

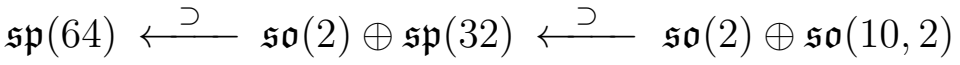

$$
\begin{aligned}
& 64 \longrightarrow(2,32) \longrightarrow\left(2, S^{+}\right)=(2,32)
\end{aligned}
$$

Type IIA is also embedded into a superconformal algebra with 64 spinor charges, $\mathfrak{o} \mathfrak{s p}(1, N 2 n)=\mathfrak{o s p}(1 \mid 64)$. As before, we have

$$
\begin{aligned}
& \mathfrak{s p}(64) \stackrel{\supset}{\longleftarrow} \mathfrak{s p i n}(11,2) \stackrel{\supset}{\longleftarrow} \quad \mathfrak{s o}(10,2) \\
& 64 \longrightarrow S=64 \longrightarrow S^{+} \oplus S^{-}=32^{+} \oplus 32^{-} \text {. }
\end{aligned}
$$

The $d=4$ case $\quad$ It is interesting to consider the case of dimension 4 with all possible signatures $\rho=0,2,4$. For $\rho=4$ (Euclidean case) the superalgebra is $\mathfrak{s u}^{*}(4 \mid 2 N)$, for $\rho=2$ (Lorentzian case) the superalgebra is $\mathfrak{s u}(2,2 \mid N)$ and for $\rho=0$ it is $\mathfrak{s l}(4 \mid N)$.

The superalgebras with 32 charges are $\mathfrak{s u}^{*}(4 \mid 4), \mathfrak{s u}(2,2 \mid 4)$ and $\mathfrak{s l}(4 \mid 4)$ (since $n=m$ these algebras have no $\mathfrak{u}(1)$ or $\mathfrak{o}(1,1)$ factor). They correspond to the underlying symmetries of $N=4$ Euclidean Yang-Mills [31, 32], $N=4$ ordinary Yang-Mills and $N=4$ self dual Yang-Mills 33 considered in the literature. Only the latter two exist with eight charges, corresponding to $N=1$ supersymmetry, $\mathfrak{s u}(2,2 \mid 1)$ and $\mathfrak{s l}(4 \mid 1)$. 
We note that these minimal superconformal algebras have a further extension into orp $(1 \mid 8)$ [9, 34], since $\mathfrak{s p}(8, \mathbb{R})$ contains both, $\mathfrak{s u}(2,2) \oplus \mathfrak{u}(1)$ and $\mathfrak{s l}(4, \mathbb{R}) \oplus \mathbb{R}$. o $\mathfrak{s p}(1 \mid 8)$ can also be viewed as an anti de Sitter super algebra in $d=5$, so by contraction we get the five dimensional super Poincaré algebra with $Z$-charges. The $Z$-charges do not appear if we make the contraction from the minimal superalgebra $\mathfrak{s u}(2,2 \mid 1)$.

It is interesting to note that the enlargement of $\mathfrak{s u}(2,2) \oplus \mathfrak{u}(1)$ to $\mathfrak{s p}(8, \mathbb{R})$ does not change the rank of the algebra, so the number of quantum numbers that label an irreducible unitary representation would be the same.

$\mathfrak{o s p}(1 \mid 8)$ has (as all superconformal algebras) an $\mathfrak{o}(1,1)$ grading

$$
\mathfrak{o} \mathfrak{s p}(1 \mid 8)=\mathcal{L}^{-1} \oplus \mathcal{Q}^{-1 / 2} \oplus \mathcal{L}^{0} \oplus \mathcal{Q}^{+1 / 2} \oplus \mathcal{L}^{+1},
$$

with $\mathcal{L}^{0}=\mathfrak{s l}(4, \mathbb{R}) \oplus \mathfrak{s o}(1,1)$. Note that $\mathfrak{s l}(4, \mathbb{R})=\mathfrak{s p i n}(3,3)$ and that we have

$$
\mathfrak{s o}(3,1) \oplus \mathfrak{s o}(2) \in \mathfrak{s o}(3,3), \quad(\rho=2)
$$

with $\mathfrak{s o}(2)$ being the R-symmetry of $\mathfrak{s u}(2,2 \mid 1)$ and

$$
\mathfrak{s o}(2,2) \oplus \mathfrak{s o}(1,1) \in \mathfrak{s o}(3,3), \quad(\rho=0)
$$

with $\mathfrak{s o}(1,1)$ being the R-symmetry of $\mathfrak{s l}(4 \mid 1)$.

\section{Appendix}

In this appendix we give some definitions that are used throughout the paper. There are many references where these concepts are treated in great detail. We cite here the ones we have used [35, 36, 3, 37].

A. A super Lie algebra is a $\mathbb{Z}_{2}$-graded vector space $\mathfrak{g}=\mathfrak{g}_{o}+\mathfrak{g}_{1}$ with a bilinear operation $[]:, \mathfrak{g} \times \mathfrak{g} \rightarrow \mathfrak{g}$ satisfying the following properties:

a. We say that an element $a \in \mathfrak{g}$ is homogeneous of degree $p_{a}=0,1$ if $a \in \mathfrak{g}_{0}$ (a is even) or $a \in \mathfrak{g}_{1}$ (a is odd) respectively. We then have that for $a$ and $b$ homogeneous elements of $\mathfrak{g}$

$$
p_{[a, b]}=p_{a}+p_{b} \quad \text { modulo } \mathbb{Z}_{2} .
$$

b. The bracket is graded-skew symmetric,

$$
[a, b]=-(-1)^{p_{a} \cdot p_{b}}[b, a],
$$


with $a$ and $b$ homogeneous in $\mathfrak{g}$.

c. Generalized Jacobi identity,

$$
(-1)^{p_{a} \cdot p_{c}}[a,[b, c]]+(-1)^{p_{c} \cdot p_{b}}[c,[a, b]]+(-1)^{p_{b} \cdot p_{a}}[b,[c, a]]=0
$$

if $a, b$ and $c$ are homogeneous in $\mathfrak{g}$.

It follows that $\mathfrak{g}_{0}$ is an ordinary Lie algebra and that the subspace of the odd elements $\mathfrak{g}_{1}$ carries a representation of $\mathfrak{g}_{0}$.

A superalgebra $\mathfrak{g}$ is simple if it has no other ideal than 0 and $\mathfrak{g}$. If $\mathfrak{g}$ is simple, then the representation of $\mathfrak{g}_{0}$ on $\mathfrak{g}_{1}$ is faithful and $\left[\mathfrak{g}_{1}, \mathfrak{g}_{1}\right]=\mathfrak{g}_{0}$. If these two conditions are satisfied and, in addition the representation of $\mathfrak{g}_{0}$ on $\mathfrak{g}_{1}$ is irreducible, then $\mathfrak{g}$ is simple.

B. We give here the definition of some classical complex superalgebras that are used in the text.

Let $V=V_{0} \oplus V_{1}$ be a $\mathbb{Z}_{2}$ graded vector space over $\mathbb{C}$ with $\operatorname{dim} V_{0}=m$ and $\operatorname{dim} V_{1}=n$. Then we have that the endomorphisms of $V$ are also a graded vector space. In terms of a basis

$$
\begin{gathered}
\operatorname{End}(V)=\left\{\left(\begin{array}{cc}
A_{m \times m} & B_{n \times m} \\
C_{m \times n} & D_{n \times n}
\end{array}\right)\right\}, \\
\operatorname{End}(V)_{0}=\left\{\left(\begin{array}{cc}
A_{m \times m} & 0 \\
0 & D_{n \times n}
\end{array}\right)\right\}, \quad \operatorname{End}\left(V_{1}\right)=\left\{\left(\begin{array}{cc}
0 & B_{n \times m} \\
C_{m \times n} & 0
\end{array}\right)\right\} .
\end{gathered}
$$

it is endowed with a super Lie algebra structure with bracket

$$
[a, b]=a b-(-1)^{p_{a} p_{b}} b a .
$$

This superalgebra is denoted by $\mathfrak{g l}(m \mid n)$. We define the supertrace of an element of $\mathfrak{g l}(m, n)$ as

$$
\operatorname{str}\left(\begin{array}{ll}
A & B \\
C & D
\end{array}\right)=\operatorname{tr}(A)-\operatorname{tr}(B) .
$$

The subspace of elements of $\mathfrak{g l}(m, n, \mathbb{C})$ that have zero supertrace is a subsuperalgebra denoted by $\mathfrak{s l}(m, n, \mathbb{C})$. If $m \neq n$, then $\mathfrak{s l}(m \mid n, \mathbb{C})$ is a simple superalgebra. Its even part is $\mathfrak{s l}(m, \mathbb{C}) \oplus \mathfrak{s l}(n, \mathbb{C}) \oplus \mathbb{C}$. 
In $\mathfrak{s l}(n, n)$ there is a one dimensional ideal $\mathfrak{i}$ generated by the matrix $\mathbb{1}_{2 n \times 2 n}$. The algebra $\mathfrak{s l}(n \mid n) / \mathfrak{i}$ is also simple. Its even part is $\mathfrak{s l}(n, \mathbb{C}) \oplus$ $\mathfrak{s l}(n, \mathbb{C})$.

Let $F$ be a non degenerate bilinear form on the graded vector space $V$.We assume that it is graded symmetric, that is, $F(a, b)=(-1)^{p_{a} p_{b}} F(b, a)$. This means that the restriction to $V_{0}$ is symmetric and the restriction to $V_{1}$ is antisymmetric. We assume also that $F(a, b)=0$ if $a \in V_{0}$ and $b \in V_{1}$, so $V_{0}$ and $V_{1}$ are orthogonal. Because of the non degeneracy, we have that $\operatorname{dim}\left(V_{1}\right)$ must be an even number. In a certain basis the bilinear for is given by a matrix

$$
\left(\begin{array}{cc}
\mathbb{1}_{m \times m} & 0 \\
0 & \Omega_{2 p \times 2 p}
\end{array}\right), \quad \Omega_{2 p \times 2 p}=\left(\begin{array}{cc}
0 & I \\
-I & 0
\end{array}\right) .
$$

The subspace of $\mathfrak{g l}(m \mid 2 p, \mathbb{C})$ which satisfies

$$
a^{t} F+F a=0, \quad a^{t}=\left(\begin{array}{cc}
A^{T} & C^{T} \\
-B^{T} & D^{T}
\end{array}\right)
$$

( $T$ denotes the usual transpose) is a simple Lie superalgebra whose even part is $\mathfrak{s o}(m, \mathbb{C}) \otimes \mathfrak{s p}(2 p, \mathbb{C})$. It is called the ortosymplectic algebra, $\mathfrak{o} \mathfrak{s p}(m \mid 2 p)$.

C. The complex Lie superagebras defined above have real forms that are real simple Lie superalgebras. These real forms are determined by the real form of the even part (see Refs. [30, 9, 10]). We list here the ones that are of interest for our paper. The notation that we use for the real forms of Lie algebras is the standard one [38].

Real forms of $\mathfrak{s l}(m \mid n, \mathbb{C})$.

1. $\mathfrak{s l}(m \mid n, \mathbb{R})$, with even part $\mathfrak{s l}(m, \mathbb{R}) \oplus \mathfrak{s l}(n, \mathbb{R}) \oplus \mathbb{R}$.

2. $\mathfrak{s u}(m \mid n)$, with even part $\mathfrak{s u}(m) \oplus \mathfrak{s u}(n) \oplus \mathfrak{u}(1)$.

3. $\mathfrak{s u}(m, n \mid p, q)$, with even part $\mathfrak{s u}(m, n) \oplus \mathfrak{s u}(p, q) \oplus \mathfrak{u}(1)$.

4. $\mathfrak{s u}^{*}(m \mid n)$, with even part $\mathfrak{s u}^{*}(m) \oplus \mathfrak{s} \mathfrak{u}^{*}(n) \oplus \mathfrak{s o}(1,1)$.

Real forms of $\mathfrak{o s p}(m \mid n, \mathbb{C})(n=2 p)$.

1. osp $(m \mid n, \mathbb{R})$, with even part $\mathfrak{s o}(m, \mathbb{R}) \oplus \mathfrak{s p}(n, \mathbb{R})$.

2. $\mathfrak{o} \mathfrak{s p}(m, q \mid n)$, with even part $\mathfrak{s o}(m, q) \oplus \mathfrak{s p}(n)$.

3. $\mathfrak{o s p}\left(m^{*} \mid 2 s, 2 t\right)$, with even part $\mathfrak{s o}^{*}(m) \oplus \mathfrak{u} \mathfrak{s p}(2 s, 2 t)$. 
Additionally, there are other simple Lie superalgebras that are constructed by taking the complex Lie superalgebra and looking at it as a real Lie superalgebra space of twice the dimension. Their even parts correspond to the complex even parts taken as real Lie algebras. We denote those by $\mathfrak{s l}(n \mid m, \mathbb{C})_{\mathbb{R}}$ and $\mathfrak{o s p}(n \mid m, \mathbb{C})_{\mathbb{R}}$.

\section{Acknowledgements}

S. F. would like to thank the Dipartimento di Fisica, Politecnico di Torino for its kind hospitality during the completion of this work. The work of S. F. has been supported in part by the European Commission RTN network HPRN-CT-2000-00131, (Laboratori Nazionali di Frascati, INFN) and by the D.O.E. grant DE-FG03-91ER40662, Task C. M. A. Ll. would like to thank the Department of Physics and Astronomy of the University of California, Los Angeles for its hospitality during the completion of this work.

\section{References}

[1] J. Wess and B. Zumino, "Supergauge transformations in four dimensions", Nucl. Phys B70 39 (1974).

[2] W. Nahm, V. Rittenberg and M. Scheunert, "Classification Of All Simple Graded Lie Algebras Whose Lie Algebra Is Reductive", J. Math. Phys. 171626 (1976).

[3] V. G. Kac, "A Sketch of Lie Superalgebra Theory", Commun. Math. Phys. 5331 (1977); V. G. Kac, "Lie Superalgebras", Adv. Math. 268 (1977); V. G. Kac, J. Math. Phys. 21689 (1980).

[4] I. Bars and M. Günaydin, "Construction of Lie Algebras and Lie Superalgebras from Ternary Algebras", J. Math. Phys. 20(9) 1977 (1979).

[5] J. Strathdee, "Extended Poincare Supersymmetry", Int. J. Mod. Phys. A2 (1) 273 (1987).

[6] D. Alekseevsky and V. Cortés, "Classification of N-(super)-extended Poincaré algebras and bilinear invariants of the spinor representation of $\operatorname{Spin}(p, q)$ ". Commun. Math. Phys. 183, 477-510 (1997). 
[7] M. J. Duff, R. R. Khuri and J. X. Lu, "String Solitons", Phys. Rept. 259, 213 (1995).

[8] G. W. Gibbons and P. K. Townsend, "Vacuum Interpolation In Supergravity Via Super P-Branes", Phys. Rev. Lett. 713754 (1993).

[9] R. D'Auria, S. Ferrara, M. A. Lledó and V. S. Varadarajan, "Spinor Algebras," J. of Geom. and Phys. 40 101-129 (2001).

[10] R. D'Auria, S. Ferrara and M. A. Lledó , "On the Embedding of SpaceTime Symmetries into Simple Superalgebras," Lett. Math. Phys. 57 (2001) 123-133.

[11] W. Nahm, "Supersymmetries and their Representations", Nucl. Phys. B 135149 (1978).

[12] S. Coleman, J. Mandula, "All Possible Symmetries of the S Matrix", Phys. Rev. 1591251 (1967).

[13] R. Haag, J. Lopuszański and M. Sohnius, "All Possible Generators of Supersymmetries of the S Matrix", Nucl. Phys. B88 257 (1975).

[14] E. Witten, "Five-branes and M-theory on an Orbifold," Nucl. Phys. B 463, 383 (1996).

[15] C. Vafa, "Evidence for F-Theory," Nucl. Phys. B 469, 403 (1996).

[16] I. Bars, "Two-Time Physics in Field Theory," Phys. Rev. D62 046007 (2000); "A case for 14 dimensions," Phys. Lett. B403 257-264 (1997); "S-Theory," Phys. Rev. D55 2373-2381 (1997).

[17] P.K. Townsend, "M(embrane) theory on $T^{9}, "$ Nucl. Phys. Proc. Suppl. 6811 (1998).

[18] P.K. Townsend, "p-Brane Democracy", in the proceedings of the March 95 PASCOS/Johns Hopkins conference. hep-th/9507048.

[19] P. Horava, "M-theory as a Holographic Field Theory," Phys. Rev. D 59 046004 (1999). 
[20] E. Bergshoeff and A.Van Proeyen, "The Many Faces of $\operatorname{OSp}(1 \mid 32), "$ Class. Quant. Grav. 17, 3277 (2000); "Symmetries of string, M and F-theories," Class. Quant. Grav. 18, 3083 (2001); "The unifying superalgebra $\operatorname{OSp}(1 \mid 32), "$ hep-th/0010194.

[21] R. D'Auria and P. Frè, "Geometric Supergravity in $D=11$ and its Hidden Supergroup", Nucl. Phys. B 201101 (1982).

[22] E. Angelopoulos, M. Flato, C. Fronsdal and D. Sternheimer, "Massless Particles, Conformal Group And De Sitter Universe", Phys. Rev. D 23 (1981) 1278.

[23] M. Günaydin, "Unitary Supermultiplets of $\operatorname{OSp}(1 / 32, \mathrm{R})$ and M-theory", Nucl. Phys. B 528432 (1998).

[24] P. West, "Hidden Superconformal Symmetry in M theory," JHEP 0008 007 (2000).

[25] O. Aharony, S. S. Gubser, J. Maldacena, H. Ooguri and Y. Oz, "Large N Field Theories, String Theory and Gravity". Phys. Rept. 323, 183 (2000).

[26] E. Witten, "Quantum Gravity in de Sitter Space," arXiv:hepth/0106109.

[27] A. Strominger, "The dS/CFT Correspondence", JHEP 0110, 034 (2001).

[28] C. M. Hull, "De Sitter Space in Supergravity and M Theory", JHEP 0111, 012 (2001).

[29] C. Hull, "Duality and the signature of space-time." JHEP 9811, 017 (1998); "Symmetries and Compactifications of $(4,0)$ Conformal Gravity," JHEP 0012007 (2000).

[30] A. Van Proeyen, "Tools for supersymmetry", hep-th/9910030.

[31] D. G. McKeon, "Harmonic Superspace with Four-Dimensional Euclidean Space". Can. J. Phys. 78261 (2000); "The Simplest Superalgebras in Two, Three, Four and Five Dimensions". Nucl. Phys. B591 591 (2000). 
D. G. McKeon and T. N. Sherry, "Extended Supersymmetry in FourDimensional Euclidean Space". Annals Phys. 285221 (2000).

F. T. Brandt, D. G. McKeon and T. N. Sherry, "Supersymmetry in $2+$ 2 Dimensions". Mod. Phys. Lett. A 151349 (2000).

[32] A. V. Belitsky, S. Vandoren and P. van Nieuwenhuizen, "Instantons, Euclidean Supersymmetry and Wick Rotations". Phys. Lett. B 477335 (2000).

[33] W. Siegel, "The $\mathrm{N}=2$ (4) String is Selfdual N=4 Yang-Mills". Phys. Rev. D 463235 (1992); "Selfdual $\mathrm{N}=8$ Supergravity as Closed $\mathrm{N}=2$ (N=4) Strings". Phys. Rev. D 472504 (1993); "Supermulti-Instantons in Conformal Chiral Superspace". Phys. Rev. D 521042 (1995).

[34] J.W. Van Holten and A. Van Proeyen, "N=1 Supersymmetry Algebras in $D=2, D=3, D=4 \bmod (8) "$. J. Phys. A 153763 (1982).

[35] F. A. Berezin, "Introduction to Superanalysis". D. Reidel Publishing Company, Kluwer Academic Publishers Group (1987).

[36] B. Kostant, Differential Geometrical Methods in Mathematical Physics (Proc. Sympos., Univ. Bonn, Bonn, 1975), pp. 177-306. Lecture Notes in Math., Vol. 570, Springer, Berlin, (1977).

[37] D. A. Leites, "Introduction to the Theory of Supermanifolds". Russian Math. Surveys35:1 1-64 (1980).

[38] S. Helgason, Differential Geometry, Lie Groups and Symmetric Spaces. Academic Press, (1978). 